\title{
Phase Transformations in Nanoscale Si-Au, Ge-Au and SiGe-Au Systems
}

\author{
B. J. Kim*, C.-Y. Wen*, J. Tersoff**, L. Marlor*, S. Kodambaka***, M. C. Reuter**, E. A. Stach* \\ and F. M. Ross** \\ *Purdue University, Department of Materials Science and Engineering, West Lafayette, IN 47907 \\ **IBM T. J. Watson Research Center, Yorktown Heights, NY 10598 \\ ***University of California Los Angeles, Department of Materials Science and Engineering, Los \\ Angeles, CA 90024
}

The effect of size on phase stability and phase transformations is of both fundamental and applied interest. For example, during the nucleation and growth of self-assembled nanowires from nanoscale metal catalysts, the phase of the catalyst determines properties such as the growth rate and the structure of the nanowire. Any size-dependent or growth-rate dependent changes in the catalyst may thus have strong effects on the structures that form. These effects may be especially significant when growing nanowires of complex structure, such as axial heterostructures [1].

Based on our interest in $\mathrm{Si}$ and $\mathrm{Ge}$ nanowires catalyzed using $\mathrm{Au}$, we have therefore examined phase stability in the $\mathrm{Si}-\mathrm{Au}, \mathrm{Ge}-\mathrm{Au}$ and $\mathrm{SiGe}-\mathrm{Au}$ systems. Observations were made in situ in an ultra high vacuum transmission electron microscope that has gas delivery capabilities. We start with either size-selected aerosol particles or with evaporated and agglomerated Au films on electron transparent $\mathrm{SiN}$ membranes. The samples are heated by direct current, and $\mathrm{Si}$ or Ge is supplied by flowing the chemical vapor deposition precursor gases disilane or digermane through a capillary into the polepiece region. We use a combination of bright and dark field imaging conditions to characterize the structures present and to quantify phase stability as a function of the conditions.

FIG. 1 shows an example in which the transformation from solid Au to liquid AuSi is measured in a 45nm diameter $\mathrm{Au}$ aerosol particle [2]. This transformation takes place as Si is added to the particle, and it can be seen that the reaction proceeds from the surface inwards, so that at intermediate times the system consists of a solid Au particle surrounded by liquid AuSi. Modeling the kinetics of this transformation demonstrates that the Au-AuSi interface energy becomes important in modifying phase stability when the Au size becomes small. Analysis shows a substantial shift in the liquidus line, and a discontinuous change in the liquid composition at the transition. In addition we can extract an absolute value for the liquid-solid interfacial free energy.

On further addition of $\mathrm{Si}$, the AuSi eutectic liquid droplet becomes supersaturated with $\mathrm{Si}$ and eventually $\mathrm{Si}$ is precipitated. This nucleation event shows complex kinetics [3], as the nucleus rapidly jumps to a large volume and then grows more slowly at a rate dependent on the supply of Si. The jump size can be used to determine the supersaturation of $\mathrm{Si}$ in $\mathrm{AuSi}$ at the moment of nucleation. A comparison of kinetics at a range of temperatures, pressures and droplet sizes shows consistent behavior: supersaturation is not dependent on droplet volume, leading to predictable nucleation kinetics, which may be important in forming Si nanowires controllably.

In the Ge-Au system, we find more complex kinetics. Above the eutectic temperature, the behavior of the system is similar to that of the Au-Si system, with formation of a liquid AuGe phase followed by precipitation of Ge. However, below the eutectic temperature, a liquid phase also forms, and solid 
Ge precipitates from this liquid. We believe these observations are consistent with prior work [4], in which we observed liquid AuGe below the eutectic temperature. The liquid phase persists due to a robust supercooling that is stabilized by the supersaturation of Ge arising from the growth process.

Finally, observations of the SiGe-Au system (FIG. 2) allow us to compare supersaturation at nucleation as well as overall growth kinetics with those of pure $\mathrm{Si}$ and $\mathrm{Ge}$, so that we can examine the interaction of the two elements in the ternary eutectic system.

We believe that the application of in situ UHV TEM, which provides the opportunity to make quantitative kinetic measurements of reactions as a function of pressure, temperature and system size, can yield results that are of interest in understanding the fundamentals of phase stability as well as in controlling the formation of nanostructures for real-world applications [5].

References

[1] C.-Y. Wen, M. C. Reuter, J. Bruley, J. Tersoff, S. Kodambaka, E. A. Stach and F. M. Ross, Science 326 (2009) 1247.

[2] B. J. Kim, J. Tersoff, C.-Y. Wen, M. C. Reuter, E. A. Stach and F. M. Ross, Physical Review Letters 103 (2009) 155701.

[3] B. J. Kim, J. Tersoff, S. Kodambaka, M. C. Reuter, E. A. Stach and F. M. Ross, Science 322 (2008) 1070.

[4] S. Kodambaka, J. Tersoff, M. C. Reuter and F. M. Ross, Science 316 (2007) 729.

[5] This work was partially funded by NSF under Awards No. DMR-0606395 and DMR-0907483. We acknowledge K. B. Reuter and A. W. Ellis for their technical contributions to this project.

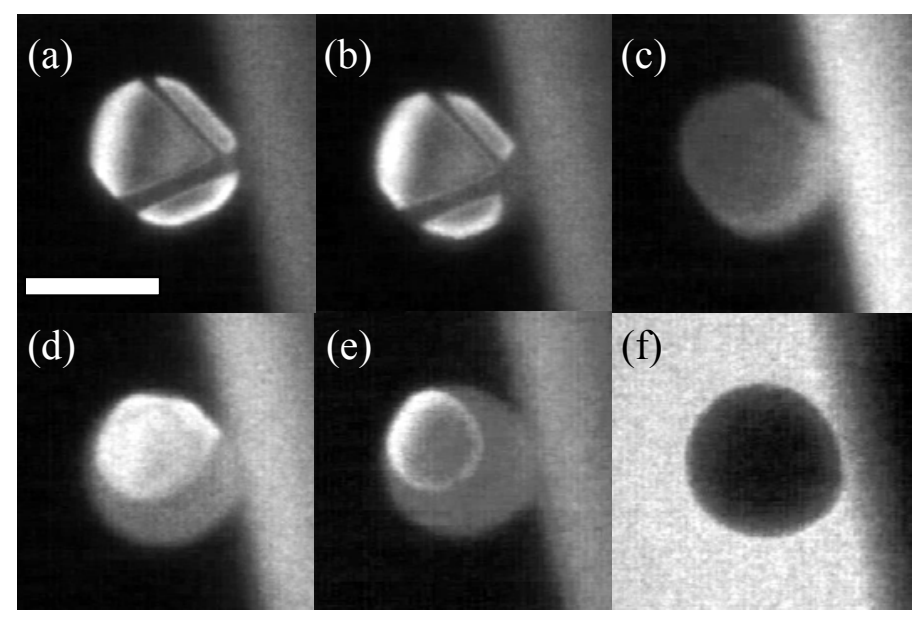

FIG. 2. The time to nucleation for $\mathrm{Si}, \mathrm{Ge}$ and $\mathrm{P}_{\mathrm{Si}}: \mathrm{P}_{\mathrm{Ge}}=$ $45: 1$ as a function of the liquid droplet radius at $470^{\circ} \mathrm{C}$ and $1 \times 10^{-7}$ Torr. The linear increase is expected from a model where the droplet collects material over its surface, but the volume has to reach a fixed composition before nucleation. The SiGe case is clearly intermediate between the pure materials.
FIG. 1. (a-e) Dark field images obtained in side view during the solid to liquid transformation at $525^{\circ} \mathrm{C}$ and $1.5 \times 10^{-6}$ Torr disilane, at $0,155,276,410$ and 444 seconds after introduction of disilane. (f) Bright field image showing the particle after the transformation is complete The scale bar is $30 \mathrm{~nm}$. The substrate appears as a vertical horizontal band at the side of each image.

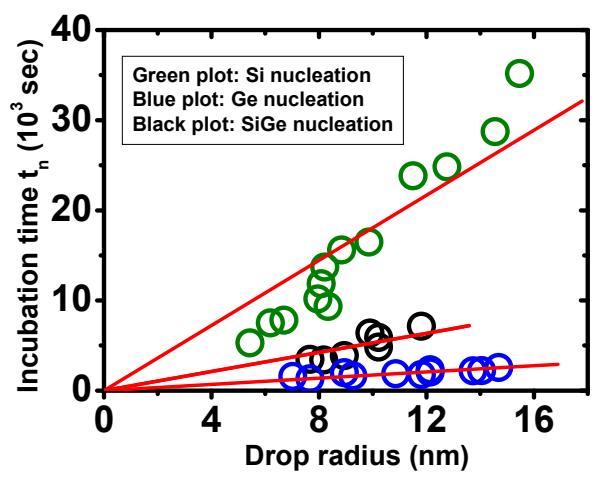

